\title{
The Sensitivity of Composite Bimodal Waveguide SU-8
}

\author{
K. GUT*
}

Department of Optoelectronics, Silesian University of Technology, Akademicka 2A, 44-100 Gliwice, Poland

This paper presents the results of measurements of the refractive index and thickness of the waveguide layer SU-8. The mode sensitivity has been calculated as a function of the thickness in a bimodal structure. The differential interference was analyzed concerning modes of the same types $\mathrm{TE}_{0}-\mathrm{TE}_{1}$ and $\mathrm{TM}_{0}-\mathrm{TM}_{1}$ and modes of the same order $\left(\mathrm{TE}_{0}-\mathrm{TM}_{0}, \mathrm{TE}_{1}-\mathrm{TM}_{1}\right)$. The thickness of the layer was determined when the interferometer is most sensitive to changes of the refractive index. It has been proved that the sensitivity of the structure can be increased by adding a nanometric layer with a high refraction index $\left(n_{\mathrm{A}}=1.975\right)$ on the waveguide layer.

DOI: 10.12693/APhysPolA.124.602

PACS: $42.25 . \mathrm{Hz}, 42.25 .-\mathrm{p}, 42.82 .-\mathrm{m}$

\section{Introduction}

The miniaturization of sources, detectors and optical system permits to apply various sensors in medicine and industry $[1,2]$. Systems of the lab-on-a-chip, recently developing, apply in their systems of detection waveguide sensors [3]. For their construction various techniques are used [4-7]. The polymer SU-8 may be used both for the construction of mechanical and waveguide structures [8]. Thanks to the rather simple technique of depositing the layers and shaping of volumetric structures the polymer SU-8 [9] is frequently applied in prototype structures MEMS and MOEMS. Because of its very good optical properties $[10,11]$ it is utilized in the production of optical sensors operating in the interferometer system [12].

SU-8 is a polymer based on epoxy resin, developed in 1989 by IBM. Thanks to its properties it is now one of the most attractive materials used in the optical planar technology. SU-8 is rather cheap and displays a high thermal and chemical stability as well as a good resistance to mechanical damages and an unusual transparency. The wide range of products ready for use, offered by manufacturers of SU-8 (MicroChem and Gersteltec Sari) permits to obtain in the course of one technological process layers with a thickness of $0.2 \mu \mathrm{m}$ up to $2 \mathrm{~mm}$.

Such good properties of the polymer SU-8 are caused by its unique structure. The chief component is epoxy resin, called $\mathrm{EPON}^{\circledR}$, consisting of $\mathrm{SU}$ monomers, and responsible for its mechanical properties and adhesion to the substrate. Another, also very important, component is photoinitiative, viz. Lewis acid, responsible for the initiation of cross-linking, in the course of which an epoxy ring is opened. The last component is a solvent, which is indispensable for warranting an adequate viscosity of the mixture.

\section{Preparation of the structure, determination of their properties}

For the purpose of investigations a series of planar waveguide structures was prepared for the SU-8 polymer of varying thicknesses. As a substrate soda-lime

\footnotetext{
*e-mail: kazimierz.gut@polsl.pl
}

glass plates were used, previously washed and rinsed in nitric acid, acetic acid and ammonia liquor. The polymer SU-8 is characterized by a weak adhesion to glass substrates [12]; therefore, in order to avoid damages of the structure in the course of depositing the polymer, the entire procedure of washing was accomplished in a laminar cell with air filtration, keeping it for 5 min in a temperature of $130^{\circ} \mathrm{C}$. Upon the substrate SU-8 was deposited by spin coating in a centrifuge from the company Rein Raum Technik Lanz, specially adapted for this purpose. The proper amount of SU-8 was batched by means of an automatic feeder with its nozzle directed towards the immovable substrate mounted in the centrifuge. The thickness of the layers depended on the velocity of gyration of the centrifuge.

In order to improve the homogeneity of the coating, each plate was after the deposition of SU-8 cooled down for five minutes to room temperature (relax time). Next, each structure was subjected to initial soft baking on a hot plate provided with a micro-processing programmer. At this stage controlling the temperature is of crucial importance for the whole process, and just therefore its precise measurement is indispensable. Each structure, irrespective of the thickness of the waveguide $\mathrm{SU}-8$ layer, was preheated from room temperature to $65^{\circ} \mathrm{C}$ with a surplus of $2{ }^{\circ} \mathrm{C}$, after which the temperature of $65^{\circ} \mathrm{C}$ was maintained for $10 \mathrm{~min}$. The following step was to heat up the structure from $65^{\circ} \mathrm{C}$ to $95^{\circ} \mathrm{C}$ with a surplus of $2{ }^{\circ} \mathrm{C} / \mathrm{min}$, which latter temperature was maintained for 60 min. After the structure had cooled down to about $30^{\circ} \mathrm{C}$, its exposure was started. For this purpose an irradiator MJB3, produced by the company Karl Suss, was used. The batching of UV radiation by means of a mercury discharge lamp (OSRAM HBO $250 \mathrm{~W}$ ) was adjusted individually for each thickness of the SU-8 layer. The irradiation was followed by post-exposure baking, similarly as in the case of preliminary soft baking. The final stage of generation was the development of the structure by means of the developer PGME [12]. After its development the structure was washed with isopropanol and dried at room temperature.

In order to determine the refractive index of a step-index waveguide structure, based on the polymer SU-8, the numerical method was applied, requiring the determi- 
nation of the effective refractive indices for each observed mode. By means of mode spectroscopy a set of effective refractive indices was determined for the wavelength $633 \mathrm{~nm}$, concerning planar waveguides. The synchronic angle was measured for the polymer waveguides obtained at rotational speeds of 2000,3000 , and $5000 \mathrm{rpm}$. Basing on the measured synchronic angle the effective refractive indices were calculated for all modes of each polarization [13].

TABLE I

Results of measurements of the refractive index and the thickness of the waveguide layers at $\lambda=633 \mathrm{~nm}$.

\begin{tabular}{c|c|c}
\hline \hline Spin speed $[\mathrm{rpm}]$ & Refractive index & Thickness $[\mu \mathrm{m}]$ \\
\hline 2000 & 1.592 & 1.38 \\
3000 & 1.597 & 1.29 \\
5000 & 1.594 & 1.20
\end{tabular}

Basing on the knowledge of the effective refractive index, the refractive index and thickness of the layer can be determined [14]. Table I contains the results of calculations.

\section{Analysis of a four-layer system}

Knowing the refractive index of the layer SU-8 and having the possibility of shaping the thickness of the layer by choosing the angular velocity of spinning, we can optimize the thickness in order to achieve the maximum sensitivity to changes in the refractive index of the cover $[15,16]$.

In the case of an interferential system the most important parameter is the mode sensitivity $S\left\{n_{\mathrm{C}}\right\}$, determining the changes of the effective refractive index $\Delta N$ due to changes of the refractive index of the cover $\Delta n_{\mathrm{C}}$ $[17,18]$ :

$$
S\left\{n_{\mathrm{C}}\right\} \cong \frac{\Delta N}{\Delta n_{\mathrm{C}}} .
$$

The differential interferometer is a simple planar waveguide, which can be realized most easily. In the waveguide two modes are excited, and a change of the refractive index of the cover involves changes of the effective refractive indices of the guided modes. The sensitivity of the differential interferometer $S_{\mathrm{D}}\left\{n_{\mathrm{C}}\right\}$ can be determined as differences of the mode sensitivity $S_{i}\left\{n_{\mathrm{C}}\right\}$ and $S_{j}\left\{n_{\mathrm{C}}\right\}$ of the guided modes [7]:

$$
\begin{aligned}
& S_{\mathrm{D}}\left\{n_{\mathrm{C}}\right\}=\frac{\Delta\left(N_{i}-N_{j}\right)}{\Delta n_{\mathrm{c}}}=\frac{\Delta N_{i}}{\Delta n_{\mathrm{c}}}-\frac{\Delta N_{j}}{\Delta n_{\mathrm{c}}} \\
& =S_{i}\left\{n_{\mathrm{C}}\right\}-S_{j}\left\{n_{\mathrm{C}}\right\} .
\end{aligned}
$$

For certain thickness of the waveguide layer structure is a bimodal structure for the polarizations TE and TM. In such a structure the modes $\mathrm{TE}_{0}, \mathrm{TE}_{1}, \mathrm{TM}_{0}, \mathrm{TM}_{1}$ can propagate. In the course of recent years the application of various order in the construction of a differential interferometer has been suggested [19-21]. Changes of the refractive index of the cover may be monitored by any arbitrary pair of modes. The possibility of monitoring the changes of the refractive index of the waveguide by analyzing the interferential field of modes of the same order was dealt with for the first time in the paper [19].

In Ref. [22] it has been shown that an additional layer of nanometer thickness with a refractive index exceeding that of the waveguide layer increases the sensitivity of the differential interferometer. In order to determine the sensitivity of the system to changes of the refractive index of the cover the four-layer system shown in Fig. 1 was analyzed.

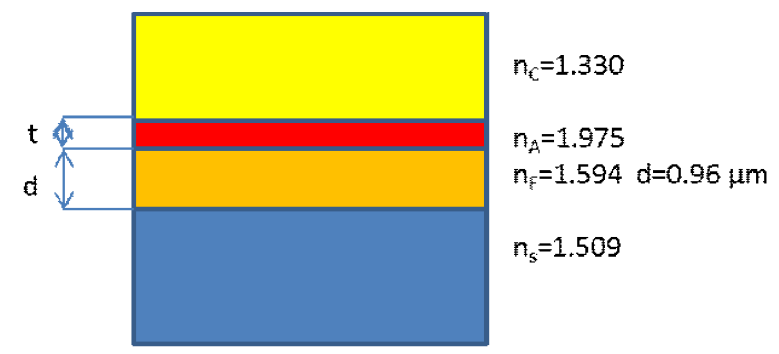

Fig. 1. Parameters of a four-layer system.

For a system with the following refractive indices: substrate $n_{\mathrm{S}}=1.509$, waveguide layer $n_{\mathrm{F}}=1.594$ and the thickness $d=0.96 \mu \mathrm{m}$ (for which the differential interferometer without additional layers applying the modes $\mathrm{TE}_{0}-\mathrm{TE}_{1}$ achieves the highest sensitivity [13]), an additional layer $n_{\mathrm{A}}=1.975$ (refractive index of the $\mathrm{ZnO}$ [6] was adopted), cover $n_{\mathrm{C}}=1.330$ were assumed in the calculations. In the calculations the wavelength was assumed to $633 \mathrm{~nm}$. The dependence of the effective refractive index as a function of the thickness $t$ of an additional layer is shown in Fig. 2.

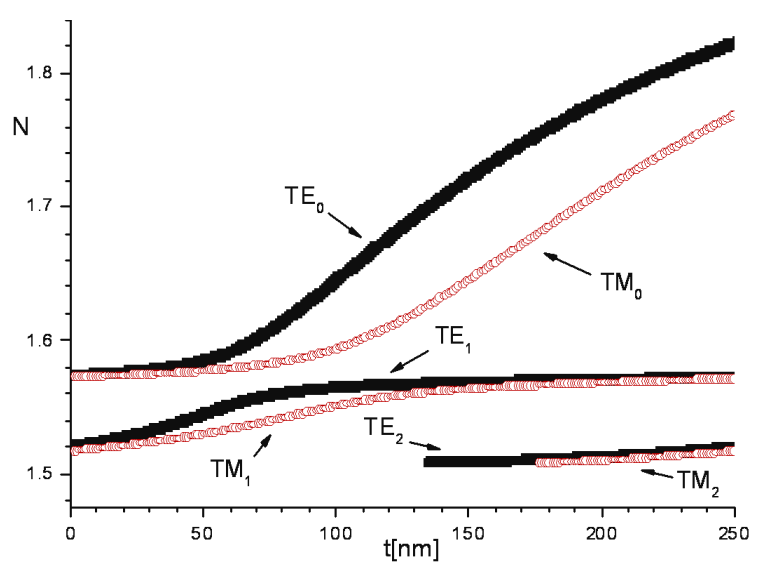

Fig. 2. Dependence of the effective refractive indices as a function of the thickness $t$ of the additional layer $t$ (SU-8 thickness of $0.96 \mu \mathrm{m}$ ).

If the thickness of the additional layer exceeds $t_{\mathrm{TE} 2}=$ $135 \mathrm{~nm}$, the system ceases to be a bimodal for TE polarization (in the case of TM polarization the corresponding value $t_{\mathrm{TM} 2}=163 \mathrm{~nm}$, for which a third mode appears). 
Making use of the definition of sensitivity (the dependence (1)) $S\left\{n_{\mathrm{C}}\right\}$ was determined as a function of the mode sensitivity concerning the thickness $t$ of the additional layer (SU-8 layer $d=0.96 \mu \mathrm{m}$ ) (Fig. 3).

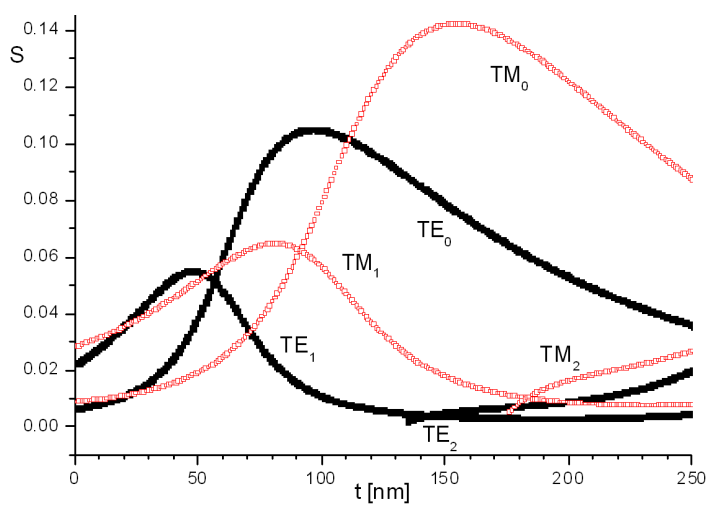

Fig. 3. Dependence of modal sensitivities as a function of the thickness $t$ of the additional layer (SU-8 thickness of $0.96 \mu \mathrm{m})$.

The modal sensitivity initially increases, reaches its maximum and then decreases with the increasing thickness of the layer. The fundamental modes of both polarizations reach a higher sensitivity than the first-order modes.

If the system operates as a differential interferometer, the difference in the sensitivity of the selected pair of modes is considered. For TE polarization based on Eq. (1), the resulting characteristics of the sensitivity of the differential interferometer $S_{\mathrm{DTE}}\left\{n_{\mathrm{C}}\right\}$ (operating on the basis of $\mathrm{TE}_{1}$ and $\mathrm{TE}_{0}$ modes) and the sensitivities of the individual modes are shown in Fig. 4.

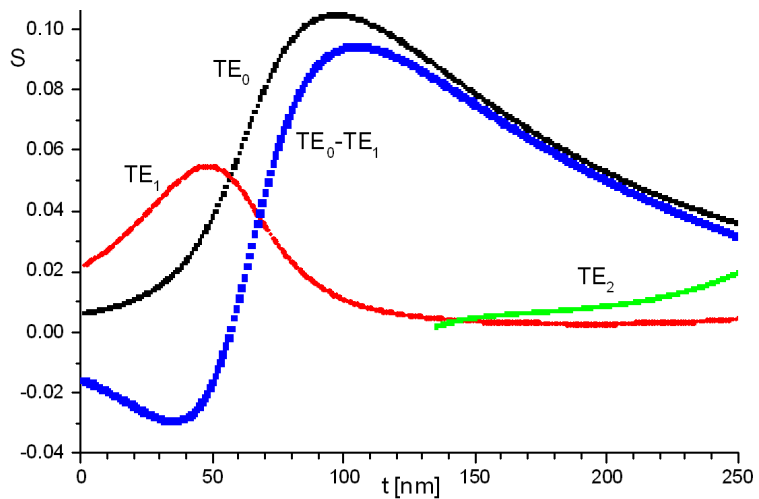

Fig. 4. Dependence of the sensitivity, the differential interferometer and of the modal sensitivity $\left(\mathrm{TE}_{0}\right.$ and $\mathrm{TE}_{1}$ ) as a function of the thickness $t$ of the additional layer (for SU-8 thickness $0.96 \mu \mathrm{m}$ ).

In the case of an additional layer thickness in the range of $0 \mathrm{~nm}$ to $50 \mathrm{~nm}$ the sensitivity of the interferometer is negative (the sensitivity $\mathrm{TE}_{1}$ mode is higher than that of the $\mathrm{TE}_{0}$ mode).
In the range above $50 \mathrm{~nm}$ the sensitivity of the interferometer increases reaching its maximum $S_{\mathrm{DTE}}\left\{n_{\mathrm{C}}\right\}=$ 0.094 at the value $t_{\text {TEmax }}=105 \mathrm{~nm}$, and then it decreases.

Figure 5 presents the characteristics of the sensitivity of the differential interferometer $S_{\mathrm{DTM}}\left\{n_{\mathrm{C}}\right\}$ applying the modes $\mathrm{TM}_{0}$ and $\mathrm{TM}_{1}$ and the sensitivity of the respective modes at the polarization TM.

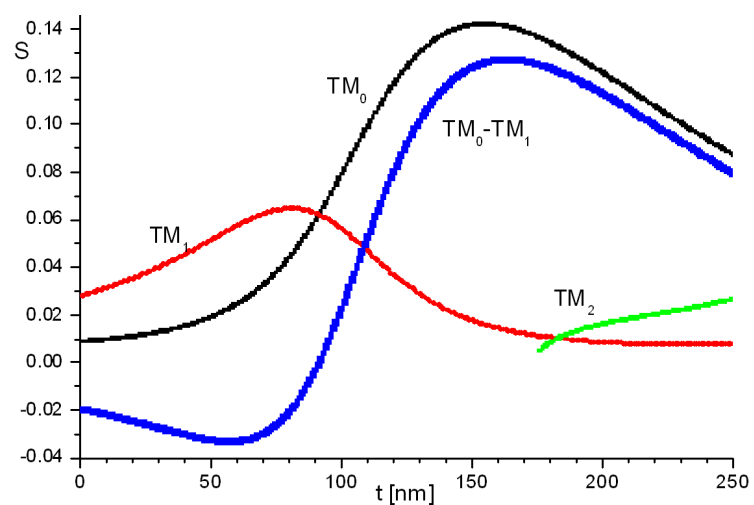

Fig. 5. Dependence of the sensitivity of the differential interferometer and the dependence of the modal sensitivities $\left(\mathrm{TM}_{0}\right.$ and $\left.\mathrm{TM}_{1}\right)$ as a function of the thickness $t$ of the additional layer (for SU-8 thickness $0.96 \mu \mathrm{m}$ ).

In the case of an additional layer thickness in the range of $0 \mathrm{~nm}$ to $80 \mathrm{~nm}$ the sensitivity of the interferometer is negative (the sensitivity of the mode $\mathrm{TM}_{1}$ is higher than that of the $\mathrm{TM}_{0}$ mode).

In the range above $80 \mathrm{~nm}$ the sensitivity of the interferometer increases reaching its maximum $S_{\mathrm{DTM}}\left\{n_{\mathrm{C}}\right\}=$ 0.127 at the values $t_{\mathrm{TMmax}}=163 \mathrm{~nm}$, and then it decreases. Table II summarizes the maximum sensitivity of the interferometer concerning a three-layer [13] and a four-layer system concerning both polarizations $\mathrm{TE}$ and TM.

TABLE II

Summary results of sensitivity of the interferometer.

\begin{tabular}{c|c|c|c|c|c}
\hline \hline \multirow{2}{*}{$\begin{array}{c}\text { Pair of } \\
\text { modes }\end{array}$} & \multicolumn{2}{|c|}{ Three-layer system [13] } & \multicolumn{2}{|c|}{$\begin{array}{c}\text { Four-layer system } \\
\left(d_{\mathrm{SU}}=960 \mathrm{~nm}\right)\end{array}$} & \begin{tabular}{c} 
Increase of \\
sensitivity \\
\cline { 2 - 5 }
\end{tabular} \\
$d[\mu \mathrm{m}]$ & $\begin{array}{c}\text { Maximum } \\
\text { sensitivity } S_{\mathrm{D} 3}\end{array}$ & $t[\mathrm{~nm}]$ & $\begin{array}{c}\text { Maximum } \\
\text { sensitivity } \\
S_{\mathrm{D} 4}\end{array}$ & $S_{\mathrm{D} 4 / S_{\mathrm{D} 3}}$ \\
\hline $\mathrm{TE}_{0}-\mathrm{TE}_{1}$ & 0.96 & 0.016 & 105 & 0.094 & $\times 5.9$ \\
$\mathrm{TM}_{0}-\mathrm{TM}_{1}$ & 1.00 & 0.019 & 163 & 0.127 & $\times 6.7$
\end{tabular}

The application of an additional layer with an adequately selected thickness can increase the sensitivity of the interferometer fivefold using the same type of TE or TM in the bimodal planar optical waveguide.

\section{Conclusion}

The investigations concern the achievement of planar waveguides on a glass substrate. For the investigations waveguides were chosen obtained at rotational speeds of 
2000,3000 , and $5000 \mathrm{rpm}$, in which two modes of polarization TE and two with TM polarization were propagated. The application of modes of the same types $\left(\mathrm{TE}_{0}-\mathrm{TE}_{1}\right.$ or $\left.\mathrm{TM}_{0}-\mathrm{TM}_{1}\right)$ permits to achieve a higher sensitivity than in the case of modes of the same orders $\left(\mathrm{TE}_{0}-\mathrm{TM}_{0}, \mathrm{TE}_{1}-\mathrm{TM}_{1}\right)$. The highest sensitivity is obtained by applying the interference of the modes $\mathrm{TM}_{0}-\mathrm{TM}_{1}$. The application with a layer of a high refractive index (1.975) and an adequately chosen thickness permits to increase the sensitivity of a double-mode differential interferometer more than fivefold.

\section{Acknowledgments}

The investigations were sponsored by the Ministry of Science and High Education of Poland within the grant O R00 017912.

\section{References}

[1] G. Konieczny, T. Pustelny, Acta Phys. Pol. A 122 , 962 (2012).

[2] K. Barczak, Bull. Pol. Acad. Sci., Techn. Sci. 59, 409 (2011).

[3] R. Walczak, P. Sniadek, J.A. Dziuban, Opt. Appl. 41, 873 (2011).

[4] A. Sabac, C. Gorecki, M. Jozwik, L. Nieradko, C. Meunier, K. Gut, J. Eur. Op. Soc-Rapid. 2, 07026 (2007).

[5] A. Opilski, R. Rogoziński, K. Gut, M. Błahut, Z. Opilski, Opto-Electron. Rev. 8, 117 (2000).

[6] P. Struk, T. Pustelny, K. Gołaszewska, E. Kaminska, M. Borysewicz, M. Ekielski, A. Piotrowska, OptoElectron. Rev. 19, 462 (2011).
[7] K. Gut, Bull. Pol. Acad. Sci., Techn. Sci. 59, 395 (2011).

[8] H. Guo, P. Zhao, G. Xiao, Z. Zhang, J. Yao, IEEE J. Sel. Top. Quant. Electron. 16, 919 (2010).

[9] K. Gut, S. Drewniak, Acta Phys. Pol. A 120, 630 (2011).

[10] K. Gut, T. Pustelny, D. Nabaglo, Acta Phys. Pol. A 118, 1136 (2010)

[11] K. Gut, D. Nabaglo, Acta Phys. Pol. A 116, 307 (2009).

[12] B. Beche, N. Pelletier, E. Gaviot, J. Zyss, Opt. Commun. 230, 91 (2004).

[13] K. Gut, Opt. Appl. 42, 407 (2012).

[14] R. Urlich, R. Torge, Appl. Opt. 12, 2901 (1973).

[15] C. Tyszkiewicz, T. Pustelny, Opt. Appl. 34, 507 (2004).

[16] R. Rogozinski, K. Gut, P. Karasinski, Z. Opilski, A. Opilski, Proc. SPIE 3581, 375 (1998).

[17] K. Gut, A. Zakrzewski, T. Pustelny, Acta Phys. Pol. A 118, 1140 (2010).

[18] K.E. Zinoviev, A.B. González-Guerrero, C. Domínguez, L.M. Lechuga, J. Lightwave Technol. 29, 1926 (2011).

[19] K. Gut, P. Karasinski, W.T. Wójcik, R. Rogozinski, Z. Opilski, A. Opilski, Opt. Appl. 29, 101 (1999).

[20] B. Pustelny, T. Pustelny, Acta Phys. Pol. A 116, 383 (2009).

[21] R. Levy, S. Ruschin, IEEE Sensors J. 9, 146 (2009).

[22] K. Gut, Acta Phys. Pol. A 114, 121 (2008). 